with it. The main known routes of transmission for HCV are parenteral, intravenous drug abuse, contaminated injection devices and receipt of unscreened blood or blood products. Objective of the study was to determine the prevalence of Hepatitis $C$ among high risk people HIV, Intra Venous Drug users (IDUs) of Eastern Nepal.

Methods The study design was descriptive cross sectional. A total of 300 samples were randomly selected from six different centres of Eastern Nepal during data collection period of one year. Structured questionnaires were used to collect demographic \& behavioural data. Venus blood was collected after taking informed consent, pretest counselling of the study subjects. Rapid Immunochromatography diagnostic kit (HCV-Tridot) was used for detection of against antibody "hepatitis C".

Results Out of total participants $95 \%$ were male and mean age was 23 years. Majority of the respondents (39\%) were adult of $20-24$ yrs age group followed by $27.7 \%$ (15-19yrs), 18\% (25-29yrs), and $13 \%$ (30-40yrs). Socio-economic status, $62 \%$ were unemployed, $23.3 \%$ labourer, $7 \%$ had different kind of business, $1.7 \%$ migrant labourer. Around 18\% participants were below poverty line.

Conclusion Prevalence of the hepatitis "C" was found to be $49 \%$ among risk group people of HIV, IDUs of Eastern Nepal. This is an alarming situation in our community, authorities of this region and country level should take action immediately to control HCV transmission as well as further prevention and treatment for HCV positives.

\section{P3.196 HIGH PREVALENCE OF HEPATITIS B (HBV) COINFECTIONS, AND LOW RATE OF EFFECTIVE HBV-VACCINATION IN MSM WITH KNOWN DATE OF HIV-1 SEROCONVERSION IN GERMANY}

doi:10.1136/sextrans-2013-051184.0653 Houareau, ${ }^{4} \mathrm{C}$ Kücherer, ${ }^{5} \mathrm{M}$ Thamm, ${ }^{4} \mathrm{~K}$ Meixenberger, $1 \mathrm{HIV}$ - the Seroconverter study group. 'HIV and STI Unit, Robert Koch-Institute, Berlin, Germany; ${ }^{2}$ German STI Society, Bochum, Germany; ${ }^{3}$ Division for Viral Gastroenteritis and Hepatitis Pathogens and Enteroviruses, Robert Koch-Institute, Berlin, Germany; ${ }^{4}$ Centre for HIV and Retrovirology, Robert Koch-Institute, Berlin, Germany; ${ }^{5}$ Central Epidemiological Laboratory, Robert Koch-Institute, Berlin, Germany

Background Men who have sex with men (MSM) with frequent sexual interactions are assumed to be at higher risk for HBV-infection. Due to partly similar transmission routes, HBV is a frequent coinfection in HIV-positive patients. HIV-coinfection accelerates disease progression of $\mathrm{HBV}$-infection resulting in earlier and more frequent occurrence of liver cirrhosis and hepatocellular carcinoma. Thus, HBV-vaccination for HIV-positive MSM is recommended in Germany.

Methods On basis of the nationwide, multicenter, open, prospective cohort study of HIV 1-seroconverters, we screened all available, yearly collected serum samples of HIV-infected MSM for anti-HBc, anti-HBs, HBs-AG, anti-HCV and Syphilis antibodies. Samples with signs of acute/chronic HBV-infection were confirmed by PCR. Prevalences were calculated on patient basis (at least one sample tested positively).

Results 5.603 samples of 1.945 MSM were screened. Median age at HIV 1-seroconversion was 33 years. In all screened MSM, prevalences of active acute/chronic HBV were $1.9 \%$, of cleared HBV $28.0 \%$. 4.8\% had signs of occult HBV-infection (solely anti-HBc positive). $48.6 \%$ were effectively vaccinated against HBV (titre > $10 \mathrm{mIU} / \mathrm{ml}$ ), of those $24.2 \%$ already at timepoint of HIV-seroconversion. Prevalences of cleared HBV and of vaccination against HBV differed significantly between age groups (table 1). $50.6 \%$ of MSM who ever had a HBV-coinfection had a further coinfection with Syphilis, 13.3\% with HCV.

Discussion Despite extensive vaccination campaigns regarding HBV-infections in MSM in Germany, less than half of all screened MSM were vaccinated. Consequentially, we found a high proportion of MSM who ever had a HBV-coinfection. Of special interest was a significant subgroup of patients having signs of occult HBVinfection, a phenomenon known to occur in immune-suppressed patients. High rates of coinfections with Syphilis and HCV in HBV/ HIV-coinfected MSM stress the demand for more extensive and tailored campaigns for HBV-vaccination and prevention of STI in general for MSM in Germany, especially for higher age groups.

\section{P3.197 UPTAKE AND CASE DETECTION OF PRENATAL SCREENING OF MATERNAL SYPHILIS, HIV AND HEPATITIS C, IN BRITISH COLUMBIA, CANADA, 2007-2011}

doi:10.1136/sextrans-2013-051184.0654

${ }^{1} M$ Gilbert, ${ }^{1} \mathrm{M}$ Alvarez, ${ }^{1} \mathrm{M}$ Krajden, ${ }^{1} \mathrm{~J}$ A Buxton, ${ }^{1} \mathrm{R}$ Lester, ${ }^{2} \mathrm{D}$ Money, ${ }^{1} \mathrm{M}$ Kuo, ${ }^{1} \mathrm{G}$ Ogilvie. 'British Columbia Centre for Disease Control, Vancouver, BC, Canada; ${ }^{2} B C$ Women's and Children's Hospital, Vancouver, BC, Canada

Background In British Columbia (BC), Canada, (pop 4.6 million), prenatal screening for rubella, syphilis, and HIV is routinely recommended while hepatitis $\mathrm{C}(\mathrm{HCV})$ screening is based on risk criteria. We determined testing uptake and case detection rates at prenatal screening for these important perinatally transmissible pathogens.

Methods We identified prenatal specimens for women aged 16-45 years between 2007-11 from the BC Public Health Reference Microbiology Laboratory and calculated the proportion of unique women screened for rubella, syphilis, HIV, and HCV per calendar year. Records were linked to laboratory surveillance data, permitting inclusion of prior testing history for HIV and HCV, to determine if detected cases were newly diagnosed at screening (new diagnoses $/ 100,000$ ) or a previously identified case. HIV and HCV prevalence were defined as all new and prior diagnoses among screened women (prevalence/100,000).

Results Of the 233,203 women who underwent one or more prenatal screening in the study period, $96.9 \%$ were screened for rubella, 93.3\% for syphilis, $93.8 \%$ for HIV, and $21.5 \%$ for HCV. Over 5 years,

Abstract P3.196 Table 1 Prevalences of acute/chronic HBV-infection, occult HBV-infection, cleared HBV-infection and HBV-vaccination in MSM with known date of HIV 1-seroconversion, by age at HIV 1-seroconversion (for comparisons: Fisher's exact test)

\begin{tabular}{|c|c|c|c|c|c|c|c|c|}
\hline & $\begin{array}{l}\text { Acute/chronic HBV: Anti-HBc }(+) \text {, } \\
\text { Anti-HBs }(-) \text {, HBs-AG }(+)\end{array}$ & $\mathbf{p}$ & $\begin{array}{l}\text { occult HBV: Anti-HBc } \\
(+) \text {, Anti-HBs (-), HBs-AG (-) }\end{array}$ & $\mathbf{p}$ & $\begin{array}{l}\text { cleared HBV: Anti-HBc (+), } \\
\text { Anti-HBs }(+) \text {, HBs-AG (-) }\end{array}$ & $\mathbf{p}$ & $\begin{array}{l}\text { HBV-vaccination: Anti-HBc (-), } \\
\text { Anti-HBs }(+) \text {, HBs-AG (-) }\end{array}$ & $\mathbf{p}$ \\
\hline Age groups & & .311 & & .053 & & $<0.001$ & & $<0.001$ \\
\hline$<25$ years $(n=256)$ & $0.4 \%$ & & $3.5 \%$ & & $14.1 \%$ & & $62.9 \%$ & \\
\hline $25-34$ years $(n=843)$ & $2.3 \%$ & & $4.0 \%$ & & $23.0 \%$ & & $51.6 \%$ & \\
\hline $35-44$ years $(n=595)$ & $2.2 \%$ & & $6.7 \%$ & & $34.6 \%$ & & $43.9 \%$ & \\
\hline $45-54$ years $(n=163)$ & $1.2 \%$ & & $3.1 \%$ & & $47.2 \%$ & & $33.1 \%$ & \\
\hline$\geq 55$ years $(n=32)$ & $0 \%$ & & $9.4 \%$ & & $50.0 \%$ & & $21.9 \%$ & \\
\hline
\end{tabular}

